\title{
Weak evidence to support benefit of periodontal maintenance therapy in prevention of tooth loss
}

\author{
Abstracted from \\ Chambrone L, Chambrone D, Lima LA, Chambrone LA. \\ Predictors of tooth loss during long-term periodontal maintenance: a systematic review of observational studies. \\ J Clin Periodontol. 2010; 37: 675-684. \\ Address for correspondence: Leandro Chambrone, Disciplina de Periodontia, \\ Faculdade de Odontologia Universidade de São Paulo, Av. Prof. Lineu Prestes, \\ 2227 Cidade Universitária, São Paulo, SP 05508-000 Brazil. E-mail: chambrone@usp.br
}

\section{Questions: What is the effect of local and systemic risk factors on tooth loss during long-term periodontal maintenance (PM)?}

Data sources Medline, Embase, Cochrane Central Register of Controlled Trials (CENTRAL), OpenSIGLE (System for Information on Grey Literature in Europe) and reference lists of identified studies. Study Selection Cohort studies, case-control studies and case series limited to patients with periodontitis who underwent active periodontal treatment (APT) and followed a maintenance care programme for at least 5 years that reported data on tooth loss were included.

Data extraction and synthesis Two independent reviewers screened the studies and extracted the data, with disagreements being resolved by discussion and consensus. Methodological quality was assessed using the Newcastle-Ottawa scale. Data were pooled into evidence tables and grouped according to the type of study. A descriptive summary was performed.

Results Thirteen retrospective case series were included in this review. The risk of bias assessment showed that eight studies were of medium methodological quality and five of low quality. Of 41404 teeth present after active periodontal treatment, 3919 were lost during PM. The percentages of tooth loss due to periodontal reasons and of patients who did not experience tooth loss varied from 1.5 to $9.8 \%$ and 36.0 to $88.5 \%$. Studies' individual outcomes showed that different patient-related factors (ie, age and smoking), and tooth-related factors (tooth type and location and the initial tooth prognosis) were associated with tooth loss during PM. Conclusions The considerable heterogeneity found among studies did not allow definitive conclusions. Age, smoking and initial tooth prognosis were found to be associated with tooth loss during PM. Overall, patients must be instructed to follow periodic PM and quit smoking (smokers). Prospective cohort studies are required to confirm the possible predictors of tooth loss due to periodontal reasons. The allocation of patients into subgroups according to the type of periodontitis and smoking frequency will allow more accurate evaluations.

\section{Commentary}

The objective of this systematic review was to examine the effect of local and systemic risk factors on tooth loss during long-term periodontal maintenance. The authors conducted a thorough search of three databases and the grey literature up to September 2009, including publications in all languages.

As the clinical question deals with prognostication, the review included observational studies of patients with chronic periodontitis, who had received active periodontal therapy followed by periodontal maintenance care for at least 5 years. Specific inclusion and exclusion criteria were outlined a priori. Thirteen retrospective case series were included and the details of each were described. No cohort or case-control studies were reported.

The major flaw in this review is the application of an adaptation of the Newcastle-Ottawa Scale (NOS) to determine risk-of-bias for included trials (http://www.ohri.ca/programs/clinical_ epidemiology/nosgen.pdf). Risk-of-bias assessment tools are used in systematic reviews in a number of ways - as a threshold for inclusion of studies; as a possible explanation for differences in results between subgroups of studies; by performing sensitivity analyses where only some of the studies are included; or by using a qualitative score as a weight in a meta-analysis of the results. However, empirical research does not support the use of these scales other than to describe the potential biases of each included trial. In fact, the Cochrane Collaboration, the group responsible for the majority of published systematic reviews in health literature, advises against the use of scales. ${ }^{1}$

The NOS assesses cohort and case-control studies and consists in terms of selection of participants (sources and selection of cases and controls), comparability of cases and controls and exposure (ascertainment of exposure and non-response rates). Thus, it was not intended for case-series. And while the face and content validity of this scale is established, criterion validity is not. Nonetheless, the authors are very clear about the limited quality of current evidence.

Furthermore, case-series studies were the best evidence available to the authors. Evidence-based dentistry is based on probabilities and rational decision-making. It is difficult to determine from caseseries, with any degree of certainty, the probability of causality and prognosis, due to the serious risk of bias by potential confounders. Thus our decision making is less dependent on evidence, relying more on a determination of the balance between risks and benefits of a particular course of action. 